\title{
Recurrent liver hydatid cyst and port site hydatid cyst in anterior abdominal wall in operated case of laparoscopic cystostomy for liver hydatid cyst: a rare complication
}

\author{
Swamy P. T.*, Jayendra G. Vagadia, Jatin G. Bhatt, Jignesh P. Dave
}

Department of Surgery, PDU Medical College, Rajkot, Gujarat, India

Received: 06 September 2021

Accepted: 11 October 2021

\section{*Correspondence:}

Dr. Swamy P. T.,

E-mail: connect2swamypt@gmail.com

Copyright: () the author(s), publisher and licensee Medip Academy. This is an open-access article distributed under the terms of the Creative Commons Attribution Non-Commercial License, which permits unrestricted non-commercial use, distribution, and reproduction in any medium, provided the original work is properly cited.

\begin{abstract}
Hydatid disease, or echinococcosis, is a widespread zoonotic parasitic disease caused by a tapeworm that continues to be a clinical and public health problem worldwide, especially in areas where animal husbandry and subsistence farming form an integral part of community life. Location of cyst in different organs of body changes the diagnostic and therapeutic management of the cyst. Four treatment options are currently available: radical surgery, conservative surgery, puncture-aspiration-injection-respiration (PAIR), and antiparasitic medical treatment. Surgery is gold standard for liver hydatid cyst and can be done by open/laparoscopic approach. We are reporting a case of recurrence of liver hydatid cyst with port site anterior abdominal wall hydatid cyst in a 40-year-old female patient operated previously for laparoscopic liver hydatid cystectomy. Port site hydatid cyst is a rare complication after laparoscopic hydatid cystotomy, but can occur due to lodgement of scolices at port site while removing daughter cyst at port site of laparoscopy.
\end{abstract}

Keywords: Echinococcosis, Hydatid cyst, Port site, Laparoscopy

\section{INTRODUCTION}

Hydatid disease (HD), or echinococcosis, is a widespread zoonotic parasitic disease caused by a tapeworm that till date continues to be a clinical and public health problem worldwide, especially in areas where animal grazing (especially sheep grazing) and subsistence farming form an integral part of community life. HD is most frequently caused by Echinococcus granulosus, and the liver is the most commonly involved organ in two thirds of patients, although it may affect any part of the body, either as a primary or secondary event. ${ }^{1}$ The liver is the most common site $(>65 \%$;), followed by the lungs $(25 \%$;); the cyst is seen less frequently in the spleen, kidneys, heart, bone, and central nervous system. ${ }^{2}$ Humans are accidental hosts and play little part within the transmission of the disease, making them so-called "dead-end hosts." HD isn't transmitted from one human host to another human. There are different treatment modalities in management of liver hydatid cyst, treatment should be considered as early as possible due to risk of complications associated with the disease if left untreated. Despite availability of non-surgical management for hydatid cyst, surgery remains mainstay treatment either through open or laparoscopic approach. The goal of surgical management of echinococcosis is to eliminate local disease, treat associated complications and avoid recurrence of the disease while minimizing morbidity and mortality related to the treatment itself. Despite surgical and medical management available for hydatid cyst treatment, recurrence remains one of the common complications encountered in surgical practice.

\section{CASE REPORT}

A 40-year-old female patient who was operated for single hydatid cyst by laparoscopic hydatid cystotomy in the year $2010(14 \times 12 \mathrm{~cm}$ cyst in right lobe of liver, touching 
diaphragm with relative thick wall, diagnosed by CECT abdomen). After 10 years she presented to outpatient department of dept. of general surgery, PDU medical college and hospital with chief complaints of lump in right upper abdomen and pain around umbilical region. On examination a spherical swelling of $8 \times 6 \mathrm{~cm}$ over right hypochondrium, which was non tender, cystic in consistency, moves with respiration. A diffuse swelling of size $5 \times 3 \mathrm{~cm}$ was palpated infraumbilical on straight leg rise test and not palpated in supine position. Ultrasonography (USG) scan of abdomen was suggestive of multiple hydatid cyst of liver largest one of $14 \times 12 \mathrm{~cm}$, and a mesenteric hydatid cyst of size $6 \times 2 \mathrm{~cm}$ around right side of umbilical region. As ultrasonography was inconclusive about multiple hydatid cyst, we proceeded with CECT of abdomen which revealed 2 hydatid cyst lesions in liver, larger one present in anterior part of right lobe of liver of size $149 \times 114 \times 112 \mathrm{~mm}$ and second one posterior to above lesion of size $104 \times 59 \times 78 \mathrm{~mm}$ which was exophytic cystic lesion, abutting to IVC and right dome of diaphragm (Figure 1). CECT of abdomen revealed that previously thought mesenteric hydatid cyst (on USG) was a hydatid cyst of size $83 \times 34 \times 39 \mathrm{~mm}$ in anterior abdominal wall of right side in right infraumbilical region which pointed more towards post laparoscopic port site hydatid cyst. Rest of biochemical and haematological investigation were normal. Patient was managed surgically, liver hydatid cyst was deroofed through right subcoastal (Kocher's) incision, with omentoplasty was done (Figure 2). The port site hydatid cystectomy was done through a small incision of $4 \mathrm{~cm}$, $1 \mathrm{~cm}$ lateral to umbilicus on right side. On exploration cystic swelling was seen with dense adhesions towards old laparoscopic scar mark and cyst was extraperitoneal, cyst was excised and site was thoroughly washed with $0.5 \%$ cetrimide solution (Figure 3 ). A single drain was kept in subhepatic region. histopathological examination of both cyst wall showed outermost peri cyst fibrous layer suggestive of hydatid cyst disease (Figure 4).

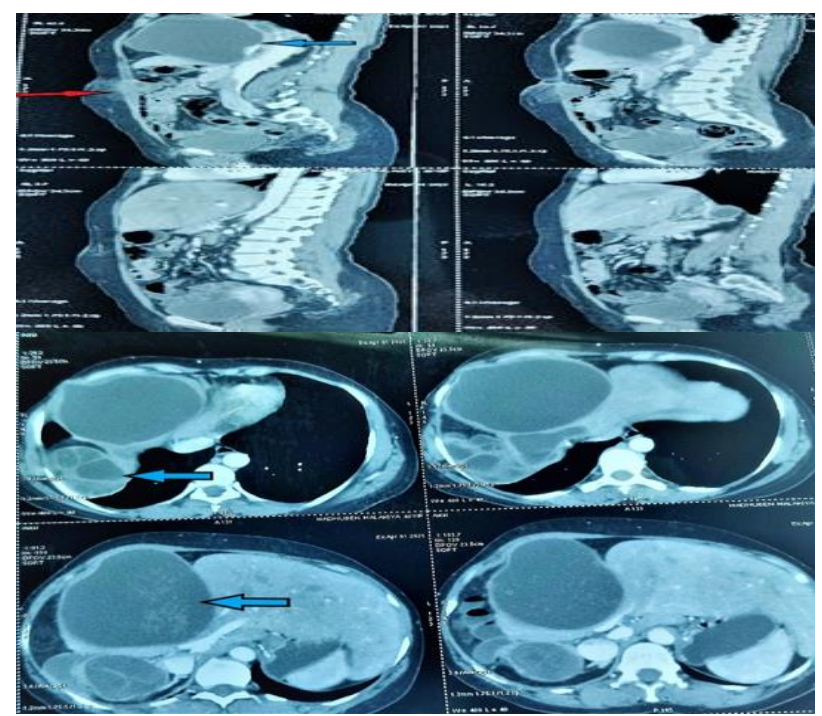

Figure 1: Port site hydatid cyst (Red arrow), liver hydatid cyst (blue arrow).

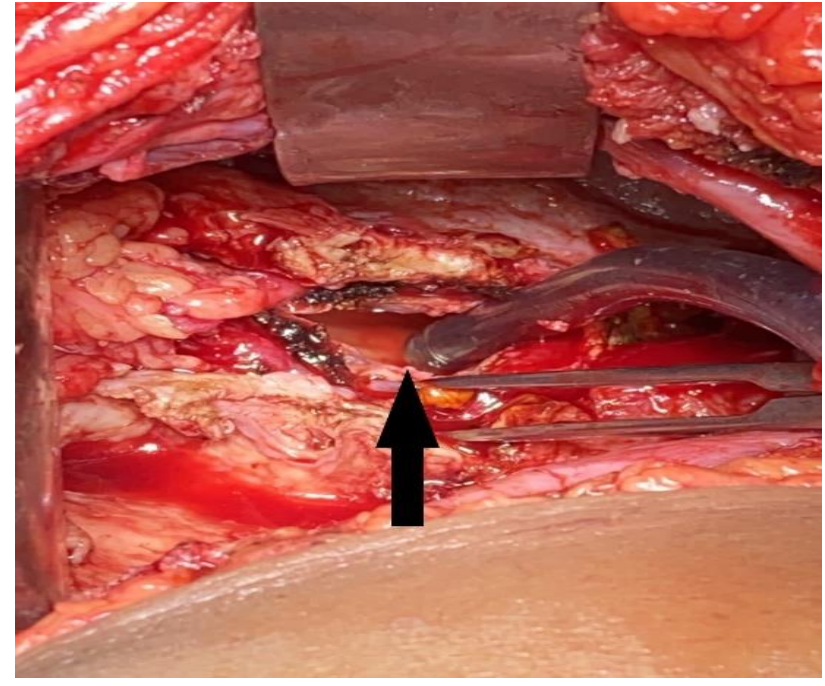

Figure 2: Intra-operative liver hydatid cyst cavity (Black arrow).

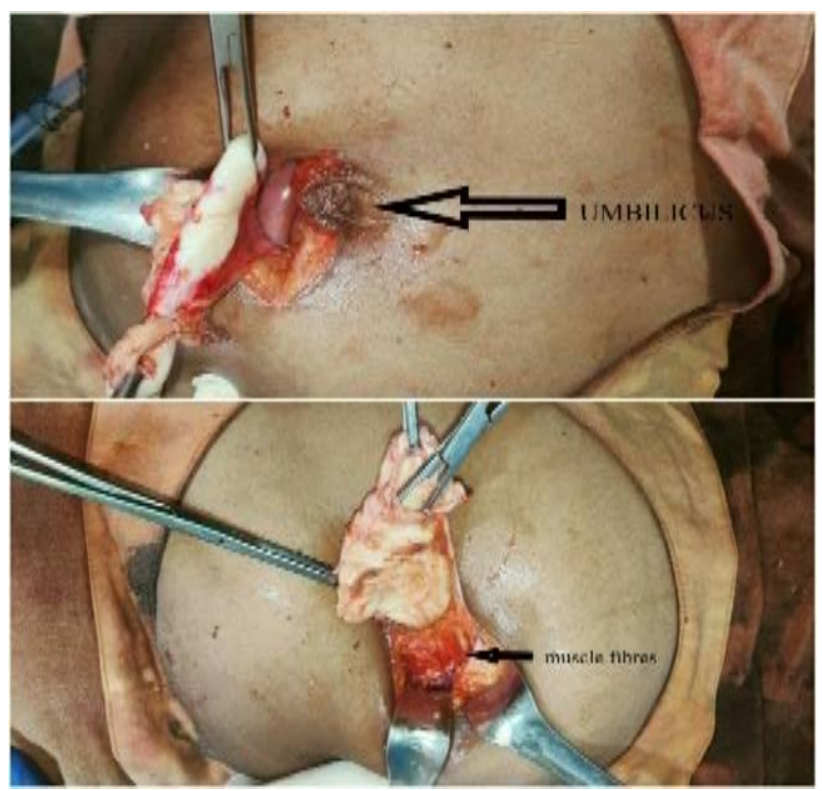

Figure 3: Intra-operative port site hydatid cyst.

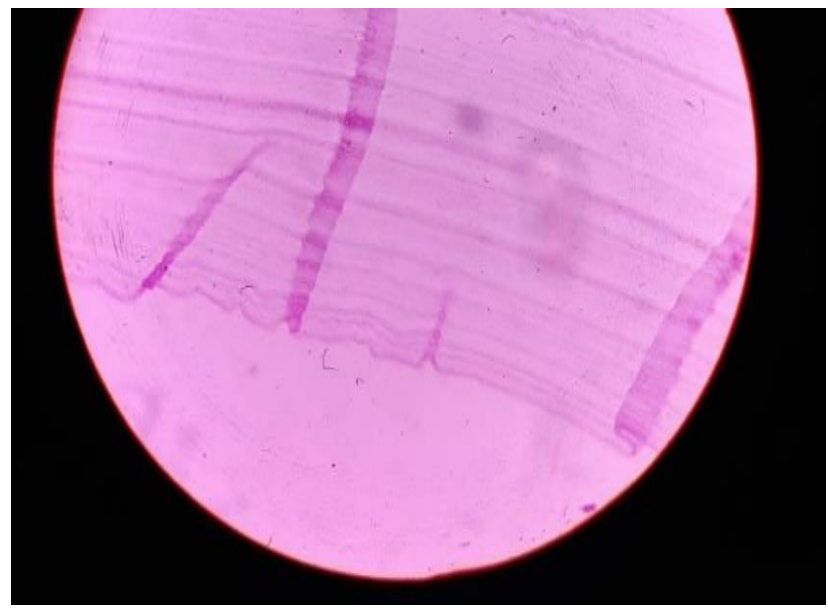

Figure 4: Histopathological image of hydatid cyst. 


\section{DISCUSSION}

The symptoms of echinococcosis vary, but consistent with cyst localization, size, type and affected organ. Although most liver hydatid cysts are asymptomatic, the most common symptom that a patient present is right upper quadrant pain and hepatomegaly. Diagnosis of echinococcosis of liver can be made easily but hydatid cyst at unusual sites (like lung, spleen, bone, kidney) needs clinical correlation, serological tests, and imaging study (USG, CT scan, and MRI scan) before any intervention can be planned. The diagnosis is usually difficult when hydatid cyst occurs at unusual locations. But USG is the preferred radiological method for cyst diagnosis because of its low cost and high specificity and sensitivity and it is easily available even at peripheral centres. CT, MRI, and MRCP have gained importance because of their ability to reveal characteristic relations of the biliary/vascular system and hydatid cyst, as well as its relations with adjacent structures. ${ }^{3}$

The objectives of the perfect treatment modality are threefold: (1) removal of the whole parasite, (2) removal of the residual cavity (cyst wall), and (3) the identification and treatment of biliary fistula. Four treatment options are currently available: radical surgery, conservative surgery, PAIR (puncture-aspirationinjection-respiration), and antiparasitic medical treatment with benzimidazoles (BMZs). ${ }^{1}$ Surgery, which is the only treatment that reaches all the three objectives of the ideal treatment modality as mentioned above, is the most efficient treatment and can be done either conventionally or laparoscopically. Percutaneous and medical treatments represent alternative methods to surgery and are associated with their own sets of drawbacks and advantages.

Sometimes surgical approach might not be feasible in patient with multiorgan hydatid cyst. The introduction of chemotherapy and PAIR of the hydatid cysts of the liver (puncture-aspiration-injection-respiration) offers an alternate treatment option, especially in inoperable patients and for cases with a high surgical risk. Cysts with homogeneously calcified cyst wall probably need no surgery but only a 'wait and observe' approach.

Many studies are conducted to check the feasibility of laparoscopic management of hydatid cyst disease. laparoscopic surgery is safe and feasible in the majority of cases, but in case of large size and intra parenchymal cyst, open surgery is more commonly performed due to technical problems encountered during laparoscopic surgery. Within the hands of an experienced surgeon complications in laparoscopic surgery decreases and is a better option, as it drastically reduces post op hospital stay and morbidity of patient. ${ }^{3}$
Despite the number of therapies now available, recurrence remains one among the main problems within the management of echinococcosis, rate of recurrence was $8.7 \%$, regardless of the type of intervention. ${ }^{4}$ The main reasons for recurrence seemed to be microscopic spillage of live parasites, failure to get rid of all viable cysts at inaccessible or difficult locations or leaving a residual cyst wall at the initial operation. But recurrence at the location of port insertion is rarely noted. Here we are reporting an operated case of liver hydatid cyst, which later on presented as recurrent liver and port site hydatid cyst which presented with vague periumbilical pain without any positive clinical findings around umbilicus. There is only one case reporting of similar occurrence of port site hydatid cyst. ${ }^{5}$ So, we are reporting this case so as to recommend to fellow surgeons to carefully wash port site with sporicidal agents to prevent port site recurrence, but there is no research work to prove the about mentioned point.

\section{CONCLUSION}

Port site hydatid cyst is a rare complication after laparoscopic hydatid cystotomy, but can occur due to lodgement of scolices at port site while removing daughter cyst through port site of laparoscopy. Despite laparoscopic deroofing of hydatid cyst is a feasible technique, proper care must be taken to prevent spillage of cystic material. If at all there is a spillage around port site thorough was must be given with sporicidal agents.

Funding: No funding sources Conflict of interest: None declared

Ethical approval: Not required

\section{REFERENCES}

1. Blumgart's Surgery of the Liver, Biliary Tract, and Pancreas, by Blumgart LH, Jarnagin WR, Elsevier. 2017;1102-21.

2. Moro P, Schantz PM. Echinococcosis: a review. Int J Infect Dis. 2009;13(2):125-33.

3. Bayrak M, Altintas Y. Current approaches in the surgical treatment of liver hydatid disease: single center experience. BMC Surg. 2019;19:95.

4. Prousalidis J, Kosmidis C, Anthimidis G, Kapoutzis K, Karamanlis E, Fachantidis E. Post-operative recurrence of cystic hydatidosis. Canadian journal of surgery. J Can de chirurgie. 2012;55(1):15-20.

5. Patel CK, Shah SM, Mehta SM, Gohil VB. Port site hydatid cyst in operated case of laparoscopic cystostomy for liver hydatid cyst: A rare complication of laparoscopic hydatid cystostomy. Int J Case Rep Images. 2016;7(9):603-5.

Cite this article as: Swamy PT, Vagadia JG, Bhatt JG, Dave JP. Recurrent liver hydatid cyst and port site hydatid cyst in anterior abdominal wall in operated case of laparoscopic cystostomy for liver hydatid cyst: a rare complication. Int Surg J 2021;8:3495-7. 